\title{
Interdependencies in Conflict Dynamics: Analyzing Endogenous Patterns in Conflict Event Data Using Relational Event Models
}

\section{Laurence Brandenberger}

\begin{abstract}
Relational event models are a powerful tool to examine how conflicts arise or manifest through human interactions and how they evolve over time. Building on event history analysis, these models combine network dependencies with temporal dynamics and allow for the analysis of group formation patternssuch as alliance or coalition formation processes-influencing dynamics or social learning. The added information on both the timing (and order) of social interactions as well as the context in which social interactions take place (i.e., the broader network in which people or actors are embedded in) can give powerful new evidence to theorized social mechanisms. This chapter provides an overview of REMs and showcases two empirical studies to illustrate the approach. The first study examines political alliance-formation patterns among countries engaging in military actions in the Gulf region. The REM shows that countries engage in military actions with other countries by balancing their relations, i.e., by supporting allies of their allies and opposing enemies of their allies. The second study shows that party family homophily guides parliamentary veto decisions and provides empirical evidence of social influencing dynamics among European parliaments.
\end{abstract}

Keywords Dynamic networks · Inferential network analysis · Conflict event data $\cdot$ Social mechanisms - Temporal dependence

\footnotetext{
L. Brandenberger $(\bowtie)$

ETH Zurich, Zurich, Switzerland

e-mail: lbrandenberger@ethz.ch
}

(C) The Author(s) 2020

E. Deutschmann et al. (eds.), Computational Conflict Research,

Computational Social Sciences, https://doi.org/10.1007/978-3-030-29333-8_4 


\section{Introduction}

Conflict has an inherently social aspect. Conflicts often arise between two parties and are often perpetrated in a broader context with the involvement of third-party actors $^{1}$ (see for instance Nelson, 1989; Crescenzi, 2003; Knoke, 1994; Wasserman and Galaskiewicz, 1994). One potential source of conflict relates to the social mechanism of social influencing and the social dynamics that build from it. Social influencing can be described as a relational process where actors modify their behavior or values to become more alike with the actors they interact with (for an overview, see Flache et al., 2017). Influencing has been described as an active force, where some actors try to persuade others to change their beliefs, attitudes, or even behavior - or as a passive force, where actors mimic values or behavior of others with whom they interact (Lindstädt et al., 2017; Shalizi and Thomas, 2011).

Social influencing can lead to conflicts within groups, as it brings some actors to do things they may not necessarily want to do (Myers, 1982; Welch and Wilkinson, 2005). Furthermore, social influencing can lead to coalition formation, where groups of actors develop their own dynamics and engage more strongly with their own group members than with other actors outside their group (Jehn et al., 2013; Berardo and Scholz, 2010). This can lead to situations where if one member of a group stands in conflict with another actor outside the group, the entire group may develop a negative relation with this outside actor. By doing so, the group reinforces their own group cohesion. Heider (1946) summarizes these coalition formation dynamics in his balance theory, where he stipulates that the enemy of my friend eventually becomes my enemy as well (see also Newcomb (1961) and Kohne et al. in the chapter "Norm Conflict in Social Networks" of this book). This indicates that conflict dynamics can go beyond dyadic relationships and a conflict between two actors can escalate into a conflict between larger groups or coalitions (Hadjikhani and Håkansson, 1996; Crano and Cooper, 1973; Labianca et al., 1998).

A question that naturally arises is: How can we detect and examine these social dynamics that can lead to conflicts in social interactions? Relational event models (REM) can be used to study multiple social mechanisms and their explanatory power of the temporal dynamics behind social interactions. REMs are inferential models that make use of temporally fine-grained records of social interactions to model complex interaction patterns and endogenous processes. REMs can be used to detect social influencing (Malang et al., 2018), understand social exchanges (Butts, 2008; Zenk and Stadtfeld, 2010; Quintane et al., 2014; Kitts et al., 2016; Stadtfeld and Geyer-Schulz, 2011), and determine causes for group or conflict formation processes (Lerner et al., 2013a; Leifeld and Brandenberger, 2019; De Nooy and Kleinnijenhuis, 2013). Building on event history analysis, REMs try to explain the occurrence of relational events. The use of the network approach allows REMs to detect complex patterns in these relational events that go beyond dyadic

\footnotetext{
${ }^{1}$ For sake of linguistic simplicity, this chapter refers to actors as a general term for different social entities, such as individuals, organizations, governments, groups, teams or other collective actors.
} 
dependencies (i.e., go beyond direct person-to-person interactions to include, for instance, the effect of third parties in these patterns) (Butts, 2008).

This chapter provides an overview over relational event models for the analysis of conflict event data. First, relational events as records of social interactions are discussed. Afterwards, REMs are presented, including how they build on event history analysis to statistically model event occurrence. The heart of REMs are endogenous network statistics that operationalize social mechanisms or patterns. The most commonly used statistics are presented in Sect.4, together with a discussion of the temporal aspects of REMs. Section 5 gives two empirical examples and discusses their operationalizations of alliance formation and social influencing. The chapter closes with a discussion of the limitations of REMs and their link to agent-based modeling through the shared use of operationalizations of social mechanisms of human interactions.

\section{Relational Events}

Conflict events often entail both a relational and directional aspect. Relational in the sense that these events report interactions among individuals, groups, or actors. These interactions are often directed from one party to another and signed. They can be negative or openly conflictive in nature and reflect opposition between two engaging parties, for instance through an act of aggression from one party directed at another party. However, they can also be positive in nature and reflect support, for instance through the exchange of information or resources. In the latter case, the absence of positive interactions may be an indication of potential conflicts among actors not sharing resources.

Alternatively, conflicts can also arise through surrounding issues and be recorded in indirect social interactions, where an active actor engages in passive issues or events. By looking at the surrounding involvement of other actors in these issues or events, a complex entanglement of actors becomes evident, where conflicts manifest themselves for instance in coalition structures and close-knit clusters of actors engaging in the same issues or events. A political debate can serve as an example here, where political actors take stances on different political issues, thus revealing their underlying coalition structure and support system (see for instance Leifeld (2017) and Hadjdinjak et al. in the chapter "Migration Policy Framing" of this book). Relational event models aim at uncovering patterns that guide these interactions and help explain how conflicts arise or manifest themselves in social interactions and how they evolve over time.

At the minimum, relational events consist of a sender node $a$, a target node $b$, and either a time stamp $t$ that records the interaction in continuous time or the place of the event in the time-ordered sequence. Once sorted in time, these events form a so-called event sequence-or event stream. Relational events can be expanded to reflect more diverse interactions. Events can be signed, for instance, to classify allegiance and opposition in international relations, friendship, and animosities in interpersonal interactions or agreement and disagreement in communication 
networks. Additionally, relational events can be weighted to reflect the intensity of the interaction. For instance, in international relations, weighted events can signify the degree of military aggression that an event encodes. In an event sequence consisting of email exchanges between colleagues in a firm, the weight of each event could correspond to the number of characters in the email or the degree of friendliness in the tone of the email. Sometimes, social interactions cannot be weighted but allow categorization. A relational event sequence can consist of different types of social interactions among sender and target nodes. For instance, in legislative politics, an event sequence can consist of members of parliament referencing (or attacking) each other in speeches, supporting each others' legislative proposal by cosponsoring them, or organizing joint press events to discuss relevant topics with the public. The assumption guiding these different types of interactions is that they co-evolve and affect each other over time.

In sum, relational event sequences are relatively flexible and are generally constrained by the data-gathering process and the degree to which social interactions can be quantified in a meaningful way.

\section{Relational Event Models}

The goal of REMs is to explain the temporal order of social interactions. Why do two people suddenly start exchanging emails? Why do two governments start engaging in military conflicts? Why do two members of parliament start collaborating with each other on a new legislative proposal? The answer to these questions is sometimes found in the broader context of these events. If two countries take up arms against each other, the alliances that form beforehand play a key role. If two people start exchanging emails it is possible that a mutual friend introduced them to each other beforehand. And if two members of parliament start working on a mutual proposal it is possible that they both learned about their mutual interest by both opposing a proposal by another member.

The events that occurred in the past often guide subsequent events and the relational event point of view can help uncover not simply how previous interactions of the two involved people or actors $a$ and $b$ shape their future interactions but also how changes in their surrounding network (i.e., with events that do not even involve $a$ or $b$ ) affect how $a$ and $b$ interact in the future. It is a powerful framework for timestamped or time-ordered analysis of social interactions that takes the surrounding context or a person's or actor's embeddedness into account. And so REMs build on event history analysis to ask the question: why does one event occur at time $t$ and has not occurred before? And in a broader sense: which patterns of past interactions can help explain a specific sequence of events?

Figure 1 depicts a simple event sequence involving four actors (=nodes). The first three recorded interactions represent support among nodes $a, b$, and $c$. After a longer break, a new node $d$ initiates a negative interaction with $b$, prompting $b$ to affirm their positive relationship with node $a$ which then brings $a$ to oppose $d$. This example already hints at the complex surroundings actors are embedded in because 


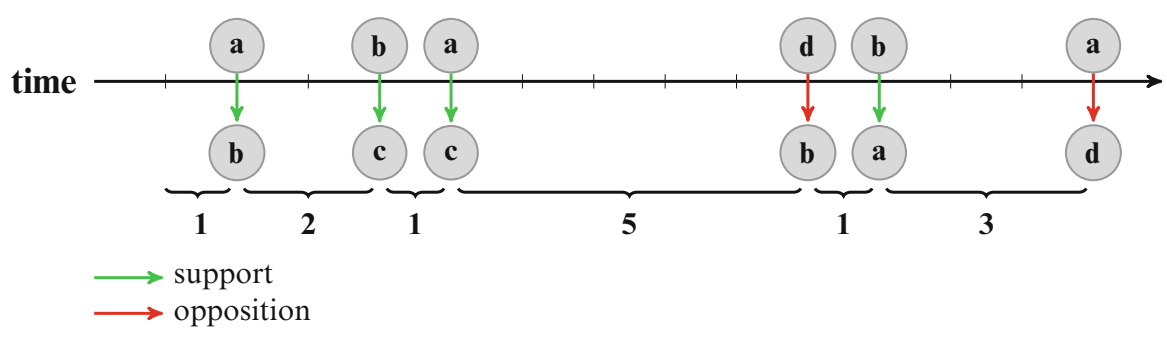

Fig. 1 Illustration of a relational event sequence depicting positive and negative interactions among four nodes $a, b, c$, and $d$

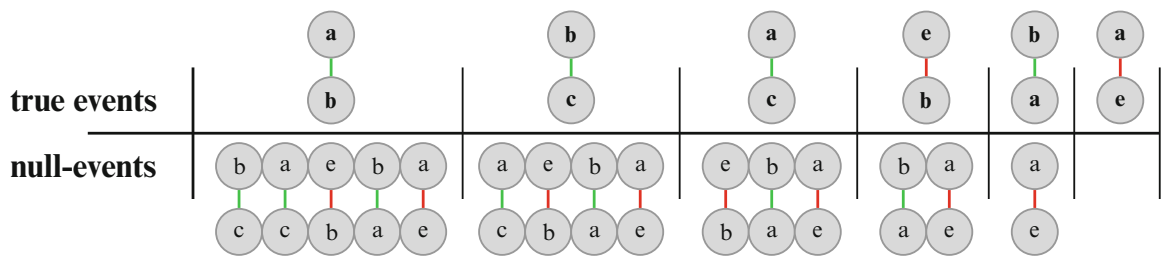

Fig. 2 Counting process data setup to estimate relational event models for the event sequence presented in Fig. 1. Each event in the event sequence forms a true event and is compared against events that could have occurred at time $t$ but did not (so-called null events). The simplest definition of the risk set for each stratum (as shown here) contains events which eventually will take place but so far have not

even though actor $d$ does not directly attack actor $a, a$ may react to an indirect threat that occurs when $d$ attacks $b$. The figure also shows the additional information that can be gained by recording events in time, as the time between events holds valuable information on how strongly future events depend on past events. The assumption that $a$ reacts to an indirect threat by $d$ is dampened a little bit by the long time it takes $a$ to oppose $d$ (4 time units in Fig. 1). The additional information on the timing of events can be used when encoding patterns, further discussed in Sect. 4.

In order to analyze the event sequence in Fig. 1, the sequence has to be transformed into so-called counting process data, first introduced by Andersen and Gill (1982). Figure 2 shows the setup of the counting process data for the event sequence presented in Fig. 1. For each unique time point in the event sequence, a stratum - or risk set-is build, containing both the true event (i.e., the event or events that occurred at time $t$ ) and null events. Null events are events that could potentially have occurred at time $t$ but did not. The simplest definition of a risk set $D$ at time $t$ contains the true events that occurred at time $t$ as well as all events that occur after time $t$. As the null events can add considerable observations to the data set, this definition of the risk set (restricted to events that occurred at one point in the event sequence) is the most sparse definition. Alternatively, if the event sequence allows for repeated events (i.e., interactions between two nodes can occur multiple times), a broader definition of the risk set may be desirable. For instance, the risk set could simply contain all possible combinations of sender and target nodes. In case the event sequence is signed and thus records both positive (e.g., supporting) 
and negative (e.g., hostile) interactions among nodes, the risk set can contain all combinations of sender and target nodes with both positive and negative edges. In the case of the event sequence presented in Fig. 1, the maximally large risk set could contain 24 events $(4 \cdot 3 \cdot 2=24 ; 4$ sender nodes times 3 remaining sender nodes (omitting self-loops) times 2 because each edge can be supporting or opposing) (see Brandenberger (2018) for additional information of risk set compositions).

Once the null events are added to the data, the hazard of event occurrence can be estimated. Standard inferential models from event history analysis can be employed because REMs assume that events are conditionally independent of one another if both exogenous and endogenous covariates are controlled for (Butts, 2008; Lerner et al., 2013b). The simplest form of the REM models event occurrence as a piecewise constant hazard model. This model assumes that the hazard (or chance) of an event occurring is constant within a time interval.

The likelihood that a specific number of events $n_{i j}(t)$ take place on a dyad $(i, j)$ within the time interval $t$ is given by the hazard rate $\lambda_{i j}(t)$, and then multiplied by the survival function $\exp \left(-\lambda_{i j}(t)\right)$, which captures all events that could have occurred at time $t$ yet did not (see Lerner et al. 2013a, pp. 18-19 and Butts 2008, pp. 161-163):

$$
\operatorname{Pr}\left(n_{i j}(t)\right)=\frac{\lambda_{i j}(t)^{n_{i j}(t)} \cdot \exp \left(-\lambda_{i j}(t)\right)}{n_{i j}(t) !} .
$$

The probability density of the event sequence $E$ can be gained by multiplying all dyads and all unique times $t_{1}$ to $t_{N}$ :

$$
f_{\lambda}\left(E ; \theta^{\lambda}\right)=\prod_{t=t_{1}}^{t_{N}}\left(\prod_{i j \in D_{\text {act }}(t)} \frac{\lambda_{i j}(t)^{n_{i j}(t)}}{n_{i j}(t) !}\right) \cdot \exp \left(-\sum_{i j \in D} \lambda_{i j}(t)\right),
$$

where $D_{\text {act }}(t)$ refers to all dyads in which at least one event occurred over $E$ and $D$ refers to all possible events that could have occurred (Lerner et al., 2013a, pp. 1819).

For continuous-time sequences, REMs use duration models or a stratified Cox model to model the time-to-next-event (for an example, see Brandenberger, 2018). In Fig. 1 these inter-event times are summarized below the curly brackets. If the exact timing of events is irrelevant or only discrete-time information is available, a stratified Cox model with constant event times can be used (Butts, 2008). The stratified Cox model estimates which factors affect event occurrence, i.e., cause an event to occur during one particular strata at time $t$, and assumes that the baseline hazard of each event is constant within a stratum but varies between strata (Cox and Oakes, 1984; Allison, 1982; Box-Steffensmeier and Jones, 2004; Allison, 2014). The stratified Cox model with constant event times can be estimated with a conditional logistic regression (Gail et al., 1980; Allison, 1982) and has become the most widely used model for REMs (Kitts et al., 2016; Quintane et al., 2014; Vu et al., 2015). In the conditional logistic regression each stratum (or risk set) compares true events, set to 1 , to null events, set to 0 . Independent and control variables are used to explain why true events occurred and null events did not. 
The standard output of a REM is comparable to outputs from logistic regressions, where for each covariate a beta-coefficient is estimated, which reflects this covariate's weight on the hazard of event occurrence. Coefficients are usually reported as log-odds and follow standard interpretations of logistic regressions.

\section{Controlling for Endogenous Network Effects}

The heart of REMs are the endogenous statistics that encode patterns in past interactions to help explain event occurrence. REMs can incorporate time-varying exogenous and endogenous variables or statistics. They are used to explain why some events take place at time $t$ and why they have not occurred before. By encoding endogenous patterns in these statistics, complex social mechanisms that guide social interactions can be uncovered. Moreover, by calculating different patterns, their effect on event occurrence can be quantified and compared to each other, illuminating which are the driving factors of social interactions.

The patterns that are encoded in these endogenous network statistics are limited only by the researchers' creativity, theoretical ideas on social mechanisms, and computational limitations (as further discussed in Sect. 5).

There are six commonly used statistics that can be expanded into more complex patterns of social interactions (see Fig. 3). Inertia measures whether events have a tendency to repeat themselves in the event sequence. Reciprocity measures whether a previous target node (node $a$ in Fig. 3) directs an event at the previous sender node $(b)$. Activity measures how active a sender node is over the course of the event sequence and popularity measures how popular a target node is. Closing triads measures whether two nodes engage with each other due to their previous engagements with a shared partner (node $b$ in Fig. 3) and four-cycles measure whether indirect engagements (nodes $a$ and $d$ in Fig. 3) drive network closure. In directed event sequences closing triads and four-cycles can be used to operationalize different closure effects (e.g., cycles or transitive triads). Inertia, activity, popularity,
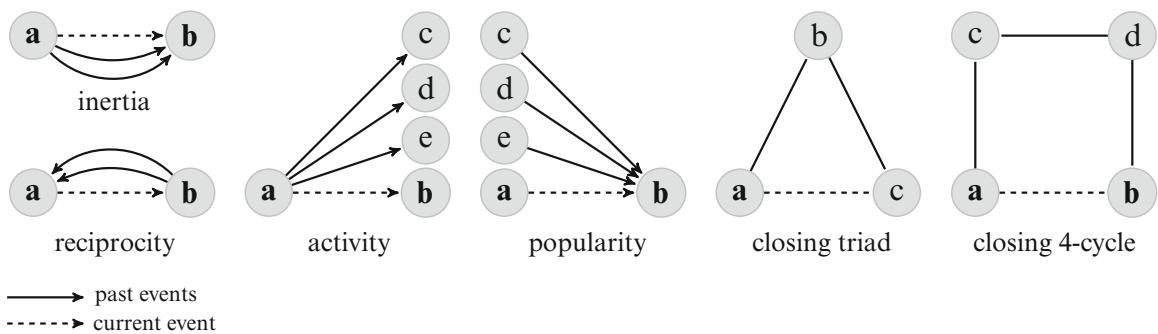

Fig. 3 Classic endogenous network effects can be used to test different interaction patterns in temporal event sequences 
and four-cycles can also be used in two-mode event sequences (where sender nodes and target nodes stem from different node sets).

In the case of country-to-country military aggression, inertia reflects repeated attacks from one country to another and reciprocity reflects whether countries have a tendency to retaliate. Activity measures whether some countries are more active in pursuing alliances or conflicts and popularity measures whether some countries are attacked (or called to an alliance) at higher rates than others. Closing triads measure whether events involving shared partners spur countries into action (for instance to defend their allies) and four-cycles measure whether there is a tendency for certain countries to remain neutral versus one another (countries $a$ and $d$ in Fig. 3).

These six statistics can be made more complex by allowing for attributes of the nodes (i.e., checking reciprocity levels among countries with the same national language) or by incorporating edge attributes (such as filtering triadic closure for positive and negative ties). The two empirical examples introduced in the next section both incorporate some of these commonly used statistics in their REM and by including edge and nodal attributes find evidence for more complex social patterns of interaction that lead to social cohesion and balance.

Another important component of endogenous network statistics is how they incorporate time. Temporal dynamics of social interactions are crucial in understanding how interactions evolve and build up over time. For relational events, each true and null event belonging to the same stratum at time $t$ (i.e., belonging to one unique point in time on the event sequence) builds a so-called network of past events $G_{t}$ to look back over the event sequence prior to time $t$ to determine whether previous events can explain which events in the stratum are true events and which are null events.

The network of past events is defined as

$$
G_{t}=G_{t}(E)=\left(A ; B ; w_{t}\right),
$$

where $E=\left(e_{1}, e_{2}, \ldots, e_{n}\right)$ represents the set of events, $A$ is the set of sender nodes, $B$ the set of target nodes (where $A=B$ for one-mode networks), and $w_{t}$ represents a weight function that can be applied to each event before time $t$.

The weight function in its simplest form gives a constant weight of 1 to each past event in $G_{t}$. However, the weight function can also be used to give events further in the past less weight than more recent events (Lerner et al., 2013a). For instance, an exponential decay function can be used to account for memory loss or forgetting:

$$
w_{t}(i, j)=\sum_{\substack{e: a_{e}=i, b_{e}=j, t_{e}<=t}}\left|w_{e}\right| \cdot e^{-\left(t-t_{e}\right) \cdot\left(\frac{\ln (2)}{T_{1 / 2}}\right)} \cdot \frac{\ln (2)}{T_{1 / 2}}
$$

where $i=a_{e} \in A$ and $j=b_{e} \in B, w_{e}$ is the weight of event $e, t$ is the current time, $t_{e}$ is the time of event $e . T_{1 / 2}$ represents the value of the half-life parameter. 
The half-life parameter specifies how fast the weight of past events diminishes, with a smaller half-life giving more weight to more recent events (Lerner et al., 2013a).

The weight function is applied to each past event that completes a social pattern or mechanism. For instance, the reciprocity statistic for the dyad $(a, b)$ counts how often an event $(b, a)$ occurred in the past:

$$
\text { reciprocity }\left(G_{t}, a, b\right)=w_{t}(b, a)
$$

For each past event $(b, a)$ the time difference $\left(t_{e}-t\right)$ to the current event dictates how much weight should be ascribed to this past event. The triadic closure statistic can be operationalized as:

$$
\text { closingTriad }\left(G_{t}, a, b\right)=\sqrt[2]{\sum_{i \in A} w_{t}(a, i) \cdot w_{t}(i, b)}
$$

This approach allows for the testing of complex social mechanisms that involve multiple people or actors and follow distinct paths.

A number of statistical tools are available to estimate REMs. They include commands to prepare the data structure of REMs, calculate the endogenous network statistics, and estimate REMs. In the statistical computing environment $\mathrm{R}$ ( $\mathrm{R}$ Core Team, 2016), the packages relevent (Butts, 2015) and rem (Brandenberger, 2019) are available to run REMs, where the latter supports exponential time weighting of past events. Alternatively, a java-based tool called eventnet (Lerner, 2019) is available for the analysis of event networks.

\section{Empirical Examples of Alliance Formation and Social Influencing}

In this section, two empirical examples are presented where alliance-building patterns and social mechanisms behind social cohesion are discussed and analyzed using relational event data.

\subsection{Military Alliance-Formation Dynamics}

Lerner et al. (2013a) model military engagements among nations involved in the Gulf region. They build on balance theory (Heider, 1946; Newcomb, 1961) to examine whether alliance-formation patterns follow the proposed patterns of interactions. Balance theory postulates that relationships among individuals or actors always have to be balanced in order to endure the passage of time. For instance in a triad (i.e., a triangular relationship pattern involving three actors), the number of positive relationships among the three actors has to be odd. 


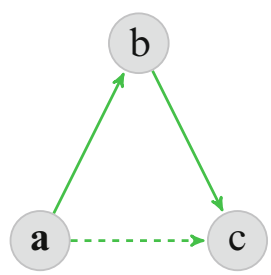

a friend of my friend becomes my friend

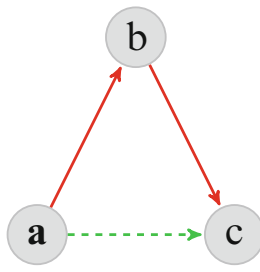

an enemy of my enemy becomes my friend

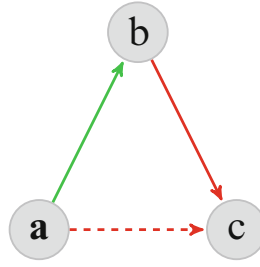

an enemy of my friend becomes my enemy

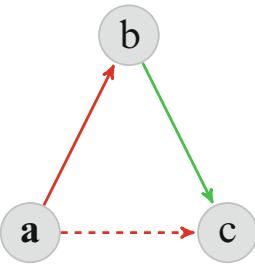

a friend of my enemy becomes my enemy

$\longrightarrow$ support

$\rightarrow$ opposition

Fig. 4 Hypotheses of balance theory: Triads are only stable if their number of positive ties is odd. The focal actor $a$ becomes active in closing the open triad (dashed relation from node $a$ to c). Lerner et al. (2013a) find strong evidence of signed triadic closure in military engagements of different nations, indicating that alliances are formed and maintained based on the mechanisms postulated by balance theory

Figure 4 shows all four possible combinations of closed triads with signed edges. All three triangles are balanced if the dyad $(a, c)$ is closed with an edge in the respected sign. Lerner et al. (2013a) use REMs to test whether nations have a tendency to close triads and if so, whether these triads are closed in the way balance theory suggests (and depicted in dashed edges between nodes $a$ and $c$ in Fig. 4).

They use data from the Kansas Event Data System (KEDS) on military actions in the Gulf region between 1979 to 1999 . The event sequence involves over 200,000 events among 168 nations (Lerner et al., 2013a, p. 7). Events are coded as one-mode network events, with nations $\times$ nations interactions encoded in time. Additionally, for each event, a weight ranging from $[-10,10]$ encodes the strength of the interaction with positive values indicating friendly interactions and support among the two nations, and negative interactions denoting military aggression. They use the eventnet application to estimate the effects of (balanced) triadic closure on event occurrence. Their results give strong support to balance theory. Moreover, they find that controlling for external alliances no longer yields any additional insights if reciprocity and triadic closure is adequately controlled for in the model (Lerner et al., 2013a, p. 28). This indicates that this form of balanced triadic closure can properly represent alliance-formation processes in international relations.

\subsection{Influencing Dynamics Among EU Parliamentary Chambers}

Malang et al. (2018) examine social influencing dynamics among national parliaments in Europe. They examine the case of the Early Warning System of the $\mathrm{EU}$, where national parliaments in Europe are allowed to veto legislative proposals 
brought forth by the European Commission. If over one third of the parliaments (or more precisely parliamentary chambers) veto the same proposal, the European Commission is forced to re-evaluate and defend the proposal. Malang et al. (2018) argue that this vetoing threshold poses an incentive for chambers to influence each other and try to get them to veto the same proposal.

They use data from 353 vetoes issued between January 2010 and September 2016 by 39 parliamentary chambers in the EU. Here, not person $\times$ person events are examined but chambers $\times$ proposals, i.e., a two-mode (or bipartite) event sequence, where sender nodes are different from target nodes and events record interactions or engagements between the two. The vetoes can be thought of as conflictual interactions of chambers with legislative proposals.

Previous studies on these vetoing actions link a chamber's decision to veto a proposal on their general attitudes towards the EU, how long they have been part of the EU, or their capacity to evaluate each proposal. However, these studies neglect the relational aspect that such collective vetoing dynamics brings with them. In order to reach the threshold, one third of all chambers have to issue a veto, but evaluating each proposal and drafting a veto takes time and resources and it is possible that chambers simplify their decision to veto by reacting to previously issued vetoes and take them as a signal to veto the proposal as well. Alternatively, it is possible that chambers try and influence each other by directly approaching members of parliament and convincing them to veto a proposal. Both explanations imply a strong social mechanism that drives vetoing behavior. One important question that arises is whether chambers influence each other based on distinct attributes they share. Do chambers with a specific attribute only react to vetoes issued by other chambers who share that attribute? And which attributes have this signaling power?

Malang et al. (2018) examine four different attributes of chambers through which this social influencing can run. They test whether two chambers are currently ruled by a party from the same party family (i.e., measuring left-right leaning similarities of chambers), whether two chambers are governed similarly and embedded in the same political system, whether two chambers joined the EU at the same time or whether the chambers are from neighboring countries. They use a popularity statistic and enhance it with chamber (or country) attribute homophily in order to test which similarities guide vetoing dynamics:

$$
\text { chamberHomophily }\left(G_{t}, a, b\right)=w_{t}(i, b)\left[a_{x}=i_{x}\right] \text {, }
$$

where $a$ refers to the focal chamber, deciding to veto a proposal $b$ and $i$ refers to other chambers that have vetoed proposal $b$ in the past and share the same attribute $x$ as the focal chamber (indicator function $\left[a_{x}=i_{x}\right]$ ) (Malang et al., 2018, p. 13).

For each of the four proposed channels of influence, they calculated the chamberHomophily-statistic. Parameter estimates for the four different operationalizations of homophily (as well as a broad range of control variables) were obtained from a conditional logistic regression on an ordinal-timed sequence of vetoes. They use the rem-package in $R$ to calculate the homophily statistics and estimate the REM. Results of the REM revealed that only two of the four 
homophily statistics had positive and significant effects on vetoing dynamics. Chambers governed by parties from the same party family and embedded in similar political systems tend to veto the same proposals (Malang et al., 2018, p. 16). Using a permutation approach on the vetoing sequence they further show that only party family similarities drive influencing dynamics because they produce event sequences with strong temporal correlation among the order of events (Malang et al., 2018, pp. 14-15).

\section{Discussion}

With increasing prowess of automated data collections, gaining access to data with higher temporal resolution of social interactions (both on- and offline) has become easier. The added temporal information in the data in turn sharpens empirical evidence and opens up new avenues of research on social interaction patterns. Social mechanisms that guide social interactions can be analyzed in much more detail and opposing hypotheses can be tested against each other.

These new avenues also pose a challenge: Many theories on social interactions do not offer clear insights into how social mechanisms can be operationalized and tested. However, the long-standing tradition of modeling dynamic behavior in agentbased models (ABM) can offer important insights. REMs can borrow interaction rules and patterns from ABMs to help operationalize different patterns in event networks. Furthermore, REMs can be used to examine the interplay of different patterns (e.g., through interaction effects of different mechanisms). Often social interactions evolve and develop over time and REMs can track these changes in behavior (for instance through temporal interaction effects) to understand how some social mechanisms evolve into others or under which circumstances (i.e., a distinct period of time, for instance an election cycle) some mechanisms dominate others. REMs can also be used to examine if social mechanisms differ between groups, as for instance shown by Brandenberger (2018) that reciprocity guides collaboration strategies of Republican members of Congress, but not Democratic members.

One limitation of REMs are computational constraints. Particularly complex (or higher-order) endogenous network statistics are challenging to compute as the calculations have to cycle through the event sequence several times to determine which past events contribute to a certain interaction pattern. This is especially difficult if event sequences are large as the network of past events $G_{t}$ becomes too extensive to filter. Sampling strategies can help alleviate this issue and reduce the computational burden. ${ }^{2}$ However it remains to be tested, which sampling strategies prove efficient for REMs in that they do not prevent the detection of complex social patterns in large and sometimes noisy social interaction data.

\footnotetext{
${ }^{2}$ Sampling strategies are often used in rare event logistic regressions (see for example King and Zeng, 2001) and could be adapted to REMs.
} 


\section{References}

Allison, P. D. (1982). Discrete-time methods for the analysis of event histories. Sociological Methodology, 13(1), 61-98.

Allison, P. D. (2014). Event history and survival analysis (2nd ed). Los Angeles: SAGE Publications.

Andersen, P. K., \& Gill, R. D. (1982). Cox's regression model for counting processes: A large sample study. The Annals of Statistics, 10, 1100-1120.

Berardo, R., \& Scholz, J. T. (2010). Self-organizing policy networks: Risk, partner selection, and cooperation in estuaries. American Journal of Political Science, 54(3), 632-649.

Box-Steffensmeier, J. M., \& Jones, B. S. (2004). Event history modeling: A guide for social scientists. Cambridge: Cambridge University Press.

Brandenberger, L. (2018). Trading favors: Examining the temporal dynamics of reciprocity in congressional collaborations using relational event models. Social Networks, 54, 238-253.

Brandenberger, L. (2019). REM: Relational Event Models. R package version 1.3.1. https://github. com/brandenberger/rem.

Butts, C. T. (2008). A relational event framework for social action. Sociological Methodology, $38(1), 155-200$.

Butts, C. T. (2015). Relevent: Relational event models. R package version 1.0-4. https://cran.rproject.org/web/packages/relevent/.

Cox, D. R., \& Oakes, D. (1984). Analysis of survival data. London: Chapman and Hall.

Crano, W. D., \& Cooper, R. E. (1973). Examination of newcomb's extension of structural balance theory. Journal of Personality and Social Psychology, 27, 344-353.

Crescenzi, M. J. (2003). Economic exit, interdependence, and conflict. The Journal of Politics, 65(3), 809-832.

De Nooy, W., \& Kleinnijenhuis, J. (2013). Polarization in the media during an election campaign: A dynamic network model predicting support and attack among political actors. Political Communication, 30(1), 117-138.

Flache, A., Mäs, M., Feliciani, T., Chattoe-Brown, E., Deffuant, G., Huet, S., \& Lorenz, J. (2017). Models of social influence: Towards the next frontiers. Journal of Artificial Societies and Social Simulation, 20(4), 31 .

Gail, M. H., Lubin, J. H., \& Rubinstein, L. V. (1980). Likelihood calculations for matched casecontrol studies and survival studies with tied death times. Biometrika, 68, 703-707.

Hadjikhani, A., \& Håkansson, H. (1996). Political actions in business networks a swedish case. International Journal of Research in Marketing, 13(5), 431-447.

Heider, F. (1946). Attitudes and cognitive organization. The Journal of Psychology, 21(1), 107-112.

Jehn, K., Rispens, S., Jonsen, K., \& Greer, L. (2013). Conflict contagion: a temporal perspective on the development of conflict within teams. International Journal of Conflict Management, 24(4), 352-373.

King, G., \& Zeng, L. (2001). Logistic regression in rare events data. Political Analysis, 9, 137-163.

Kitts, J. A., Lomi, A., Mascia, D., Pallotti, F., Quintane, E., et al. (2016). Investigating the temporal dynamics of inter-organizational exchange: Patient transfers among italian hospitals. American Journal of Sociology, 123(3), 850-910.

Knoke, D. (1994). Political networks: The structural perspective (Vol. 4). Cambridge: Cambridge University Press.

Labianca, G., Brass, D. J., \& Gray, B. (1998). Social networks and perceptions of intergroup conflict: The role of negative relationships and third parties. Academy of Management Journal, 41(1), 55-67.

Leifeld, P. (2017). Discourse network analysis: Policy debates as dynamic networks. In J. N. Victor, M. N. Lubell, \& A. H. Montgomery (Eds.), The Oxford handbook of political networks (chapter 12, pp. 301-326). Oxford: Oxford University Press. 
Leifeld, P., \& Brandenberger, L. (2019). Endogenous coalition formation in policy debates. arXiv preprint:1904.05327.

Lerner, J. (2019). Event network analyzer (eventnet): Statistical analysis of networks of relational events. Version 0.5 https://github.com/juergenlerner/eventnet.

Lerner, J., Bussmann, M., Snijders, T. A., \& Brandes, U. (2013a). Modeling frequency and type of interaction in event networks. Corvinus Journal of Sociology and Social Policy, 4(1), 3-32.

Lerner, J., Indlekofer, N., Nick, B., \& Brandes, U. (2013b). Conditional independence in dynamic networks. Journal of Mathematical Psychology, 57(6), 275-283.

Lindstädt, R., Vander Wielen, R. J., \& Green, M. (2017). Diffusion in Congress: Measuring the social dynamics of legislative behavior. Political Science Research and Methods, 5(3), 511-27.

Malang, T., Brandenberger, L., \& Leifeld, P. (2018). Networks and social influence in european legislative politics. British Journal of Political Science. https://doi.org/10.1017/ S0007123417000217.

Myers, D. G. (1982). Polarizing effects of social interaction. In Group decision making (pp. 125-161). London: Academic Press.

Nelson, R. E. (1989). The strength of strong ties: Social networks and intergroup conflict in organizations. Academy of Management Journal, 32(2), 377-401.

Newcomb, T. M. (1961). The acquaintance process as a prototype of human interaction. New York: Holt, Rinehart and Winston.

Quintane, E., Conaldi, G., Tonellato, M., \& Lomi, A. (2014). Modeling relational events. a case study on an open source software project. Organizational Research Methods, 17(1), 23-50.

$\mathrm{R}$ Core Team (2016). $R$ : A language and environment for statistical computing. Vienna: $\mathrm{R}$ Foundation for Statistical Computing. https://www.R-project.org/.

Shalizi, C. R., \& Thomas, A. C. (2011). Homophily and contagion are generically confounded in observational social network studies. Sociological Methods \& Research, 40(2), 211-39.

Stadtfeld, C., \& Geyer-Schulz, A. (2011). Analyzing event stream dynamics in two-mode networks: An exploratory analysis of private communication in a question and answer community. Social Networks, 33(4), 258-272.

Vu, D., Pattison, P., \& Robins, G. (2015). Relational event models for social learning in moocs. Social Networks, 43, 121-135.

Wasserman, S., \& Galaskiewicz, J. e. (1994). Advances in social network analysis: Research in the social and behavioral sciences. Thousand Oaks: Sage.

Welch, C., \& Wilkinson, I. (2005). Network perspectives on interfirm conflict: Reassessing a critical case in international business. Journal of Business Research, 58(2), 205-213.

Zenk, L., \& Stadtfeld, C. (2010). Dynamic organizations. how to measure evolution and change in organizations by analyzing email communication networks. Procedia-Social and Behavioral Sciences, 4, 14-25.

Open Access This chapter is licensed under the terms of the Creative Commons Attribution 4.0 International License (http://creativecommons.org/licenses/by/4.0/), which permits use, sharing, adaptation, distribution and reproduction in any medium or format, as long as you give appropriate credit to the original author(s) and the source, provide a link to the Creative Commons licence and indicate if changes were made.

The images or other third party material in this chapter are included in the chapter's Creative Commons licence, unless indicated otherwise in a credit line to the material. If material is not included in the chapter's Creative Commons licence and your intended use is not permitted by statutory regulation or exceeds the permitted use, you will need to obtain permission directly from the copyright holder.

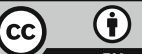

